\title{
High-fat diet-induced obesity displays altered adipocyte differentiation in the absence of Interleukin-1 Receptor I (IL-1RI)
}

\author{
K.A.J. Mitchelson ${ }^{1}$, J.C. Ralston ${ }^{1}$, C.R. Strain ${ }^{2}$, Y.M. Lenighan ${ }^{1}$, E.B. Kennedy ${ }^{1}$, G.M. Lynch ${ }^{1}$, \\ F.C. McGillicuddy ${ }^{1}$, P.W. O'Toole ${ }^{3}$ and H.M. Roche ${ }^{1}$ \\ ${ }^{1}$ Conway Institute of Biomedical and Biomolecular Research and Institute of Food and Health, University College \\ Dublin, Belfield, Dublin, Ireland, ${ }^{2}$ Teagasc Food Research Centre, Moorepark, Fermoy, Cork, Ireland and ${ }^{3}$ School of \\ Microbiology, University College Cork, Cork, Ireland.
}

High-fat diet (HFD)-induced obesity is associated with chronic low grade inflammation within adipose tissue where proinflammatory interleukin $1 \beta$ (IL1 $\beta$ ) expression is present ${ }^{(1)}$. Absence of IL-1 Receptor (IL-1RI-/-) protects against high-fat diet (HFD)-induced insulin resistance after 3 months HFD $^{(2)}$, but this protection was lost after 6 months HFD $^{(3)}$. Wildtype (WT) mice were more glucose tolerant and insulin sensitive then IL-1RI-/- mice following 6 months HFD. Also, IL-1RI-/- mice showed a loss in adipose functionality, increased adipocyte hypertrophy and reduction in TNF $\alpha$ and IL-6 secretion from stromal vascular fraction (SVF). It was unknown if/how lack of IL-1RI disrupted pre-adipocyte differentiation. Additionally, it has been suggested that microbiome transfer can affect weight gain and phenotype in in mice with inflammation-induced disease. The purpose of this study was to determine how lack of IL-1RI altered adipogenetic potential and adipokine secretion in adipose tissue.

WT and IL-1R-/- mice were fed a HFD for 6 months (45\% kcal). A subset of cages cohoused WT and IL-1R-/- mice to determine whether microbiome transfer can affect phenotype. Glucose tolerance $(1.5 \mathrm{~g} / \mathrm{kg})$ and insulin tolerance $(0.5 \mathrm{U} / \mathrm{kg})$ were tested. We investigated PPAR- $\gamma$ and FASN gene expression in preadipocytes and differentiated adipocytes, lipogenesis in preadipocytes and differentiated adipocytes and IL-6 secretion in adipose tissue organ culture in wildtype versus IL-1RI-/-. Pre-adipocytes were isolated and differentiated from adipose tissue. Adipogenic marker expression was measured by real time PCR. Oil Red O absorbance measured triacylglycerol (TAG) accumulation. IL-6 secretion from adipose tissue (AT) explants following IL-1 $\beta /$ TNF $\alpha$ stimulation was measured by ELISA. Statistical significance was determined through one - and two-way ANOVA for PCR and GTT/ ITT respectively and unpaired t-test for TAG accumulation.

IL-1RI-/- mice had increased body weight $(\mathrm{P} \leqslant 0.05)$ and AT weight compared to WT but both groups had similar glucose tolerance. IL-1RI-/- had higher insulin resistance but this was not statistically significant. Higher glucose tolerance was seen in both IL-1RI-/- and WT mice when they were cohoused together. Analysis of adipogenesis showed Fasn expression was 11- fold higher in differentiated adipocytes from IL-1RI-/- mice compared to WT mice. Conversely, Ppar- $\gamma$ expression was 37- fold higher in differentiated adipocytes from WT mice compared to IL-1RI-/- mice. TAG accumulation was $50 \%$ lower in differentiated adipocytes from IL-1RI-/- compared to WT. Adipose tissue explants had higher IL-6 secretion from WT then IL-1RI-/-. The microbiomes impact was determined and will be presented. IL-1RI-/- mice may have an increase in AT weight but adipocyte differentiation is impaired and Il-6 secretion is lower. The number of mature adipocytes and their affiliated inflammation is decreased. IL-1RI-/- may protect from HFD induced inflammation through disruption of adipogenesis. Co-housed mice total weight gain indicates a microbiome transfer which could provide a mechanism linking the beneficial alteration to adipogenesis and reduction of inflammation with a decrease in amount of adipose tissue.

This programme was primarily supported by the Irish Department of Agriculture, Food and Marine 'ImmunoMet' (Grant number: 14/F/828) programme. HMR is also the recipient of SFI principal investigator award (11/PI/1119) and the Joint Programming Healthy Life for a Healthy Diet (JPI HDHL) funded EU Food Biomarkers Alliance 'FOODBALL' (14/JP-HDHL/B3076).

1. Herder C, Dalmas E, Böni-Schnetzler M et al. (2015) Trends in Endocrinology \& Meabolism 26, 551-563.

2. McGillicuddy FC, Harford KA, Reynolds CM et al. (2011) Diabetes 60, 1688-1698.

3. McGillicuddy FC, Reynolds CM, Finucane et al. (2013) Am J Physiol Endocrinol Metab 305, E834-E844. 